\title{
A DENSITY THEOREM FOR WALSH FUNCTIONS
}

\section{J. J. PRICE}

1. Introduction. A theorem of Levinson $[3$, p. 13] on the closure of sets of exponentials contains the following result. Let $S=\left\{n_{j}\right\}$ be an increasing sequence of positive integers. Define

$$
D(S)=\limsup _{\xi \rightarrow 1-0} \limsup _{n \rightarrow \infty}(\Lambda(n)-\Lambda(\xi n)) /(n-\xi n)
$$

where $\Lambda(n)$ is the number of elements of $S$ less than $n$. Suppose $D(S)=1$. Then for each positive $\epsilon$, there is a subset $E_{0}$ of the unit interval whose measure exceeds $1-\epsilon$ and such that the family $\left[\exp \left(2 \pi i n_{j} x\right): n \equiv S\right]$ is total in $L^{2}\left(E_{\mathrm{e}}\right)$.

The proof of Levinson's theorem requires deep complex methods. In this paper, we obtain an analogous result for Walsh functions using elementary methods. Our result requires the weaker hypothesis that $\rho(S)=1$ where

$$
\rho(S)=\limsup _{k \rightarrow \infty} \limsup _{n \rightarrow \infty}(\Lambda(n+k)-\Lambda(n)) / k .
$$

It is an exercise to verify that for every sequence $S, \rho(S) \geqq D(S)$. Equality need not hold. For example, if $S$ is the sequence of all integers $n$ such that $2^{k} \leqq n<2^{k}+k$ for some $k$, then $\rho(S)=1$ but $D(S)=0$.

For definitions and properties of Walsh functions, see N. J. Fine [1].

2. Preliminaries. First some notation. All numbers mentioned will be nonnegative in tegers. The unit interval $[0,1)$ will be denoted by $I$, the dyadic interval $\left[q 2^{-p},(q+1) 2^{-p}\right)$ by $I(p, q)$. Associated with $I(p, q)$ are its characteristic functions, denoted by $\psi(p, q)$ and the Haar function supported on $I(p, q)$, denoted by $h(p, q)$. (For definition of Haar functions see [2].)

In the next section we use two results which we quote here. Both are special cases of known theorems, the first by A. A. Talalyan [5] and the second by Robert E. Zink and the author [4].

THEOREM 1. Let $\Phi$ be a sequence of real functions defined a.e. on $I$. Then $\Phi$ is total in measure on $I$ if and only if for each positive $\epsilon$ there is a subset $E_{\epsilon}$ of $I$ whose measure exceeds $1-\epsilon$ such that $\Phi$ is total in $L^{2}\left(E_{\epsilon}\right)$.

Received by the editors February 3, 1966. 
Theorem 2. Let $\Phi=\left\{\phi_{n}\right\}$ be a sequence of Haar functions. For each $n$ let $E_{n}$ be the support of $\phi_{n}$. Let $E=\lim \sup E_{n}$. Then $\Phi$ is total in measure on $I$ if and only if $I-E$ is a null set.

\section{A density theorem for Walsh functions.}

THEOREM. Let $S=\left\{n_{j}\right\}$ be an increasing sequence of positive integers and let $\Psi_{S}$ be the family of Walsh functions $\left[\psi_{n_{j}}: n_{j} \in S\right]$. If $\rho(S)=1$, then for every positive $\epsilon$ there is a subset $E_{\epsilon}$ of $I$ whose measure exceeds $1-\epsilon$ such that $\Psi_{S}$ is total in $L^{2}\left(E_{\epsilon}\right)$.

Proof. By virtue of Theorem 1, it suffices to prove $\Psi_{S}$ is total in measure on $I$. This would follow from the existence of a measure preserving transformation $T$ of $I$ on to itself that is one-to-one except for a denumerable set of points and such that the family $\Psi_{S}^{\prime}$ $=\left[\psi_{n_{j}} \circ T: n_{j} \in S\right]$ is total in measure on $I$. By virtue of Theorem 2, it suffices to show the existence of a transformation $T$ such that the linear span of $\Psi_{S}^{\prime}$ contains a family of Haar functions of the form $\left[h\left(p_{i}, q\right): 0 \leqq q<2^{p_{i}}, p_{1}<p_{2}<p_{3} \cdots\right]$.

Let $p$ be given. From the assumption $\rho(S)=1$, it follows that there is an increasing sequence $\left\{j_{i}\right\}$ such that $S$ contains all integers $n$ for which $j_{i} 2^{p} \leqq n<\left(j_{i}+1\right) 2^{p}$, or equivalently, such that

$$
\left(\Lambda\left(\left(j_{i}+1\right) 2^{p}\right)-\Lambda\left(j_{i} 2^{p}\right)\right) / 2^{p}=1 .
$$

For if $\rho(S)=1$, it is not hard to verify that also

$$
\limsup _{v \rightarrow \infty} \limsup _{u \rightarrow \infty}\left(\Lambda\left((u+v) 2^{p}\right)-\Lambda\left(u 2^{p}\right)\right) / v 2^{p}=1 .
$$

Consequently, there exist increasing sequences, $\left\{u_{i}\right\}$ and $\left\{v_{i}\right\}$ with $v_{i+1}>v_{i}+u_{i}$ such that

$$
\left(\Lambda\left(\left(u_{i}+v_{i}\right) 2^{p}\right)-\Lambda\left(u_{i} 2^{p}\right)\right) / v_{i} 2^{p}>1-2^{-p} .
$$

At least one of the quantities

$$
\left(\Lambda\left((j+1) 2^{p}\right)-\Lambda\left(j 2^{p}\right)\right) / 2^{p}, \quad v_{i} \leqq j<v_{i}+u_{i},
$$

must exceed $1-2^{-p}$; otherwise (2) could not be satisfied. But since the quantities (3) are multiples of $2^{-p}$, there exists a value $j=j_{i}$ for which (1) holds.

Let $p_{1}$ be any integer. There is an integer $j_{1}$ such that $\psi_{S}$ contains the set $\left[\psi_{j}: j=j_{1} 2^{p_{1}}+m, 0 \leqq m<2^{p_{1}}\right]$. By definition of the Walsh functions, the elements of this set have the form $\psi_{r_{1}} \psi_{m}$ where $r_{1}=j_{1} 2^{p_{1}}$. It is known ([1], p. 373) that the characteristic functions $\chi\left(p_{1}, q\right)$ are linear combinations of the functions $\left[\psi_{m}: 0 \leqq m<2^{p_{1}}\right]$. Hence, the linear span of $\Psi_{S}$ contains the set $\left[\psi_{r_{1}} \chi\left(p_{1}, q\right): 0 \leqq q<2^{p_{1}}\right]$.

For some $p_{2}$, with $p_{2}>p_{1}, \psi_{r_{1}}$ is constant on each interval $I\left(p_{2}, s\right)$. 
Each interval $I\left(p_{1}, q\right)$ is partitioned into $2^{p_{2}-p_{1}}$ intervals of the form $I\left(p_{2}, s\right)$. On half of these, $\psi_{r_{1}}$ has the value +1 ; on the others $\psi_{r_{1}}$ has the value -1 . Obviously then, the elements of this partition of $I\left(p_{1}, q\right)$ can be permuted in such a way that $c_{1} \psi_{r_{1}} \chi\left(p_{1}, q\right)$ is transformed in to the Haar function $h\left(p_{1}, q\right), c_{1}=2^{\left(p_{1}-1\right) / 2}$.

Repeating the argument given above with $p_{2}$ in place of $p_{1}$, we see that the span of $\Psi_{S}$ contains $\left[\psi_{r_{3}} \chi\left(p_{2}, q\right): 0 \leqq q<2^{p_{2}}\right]$. The function $\psi_{r_{2}}$ is constant on intervals $I\left(p_{3}, s\right)$ where $p_{3}>p_{2}$.

By induction, we obtain an increasing sequence $\left\{p_{i}\right\}$ such that for each $i$ the span of $\Psi_{S}$ contains $\left[\psi_{r_{1}} \chi\left(p_{i}, q\right): 0 \leqq q<2^{p_{i}}\right]$.

As noted earlier, there is a transformation $T_{1}$ of $I$ onto itself that permutes the intervals $I\left(p_{2}, s\right), 0 \leqq s<2^{p_{2}}$, in such a way that the intervals $I\left(p_{1}, q\right)$ are invariant and $c_{1} \psi_{r_{1}} \chi\left(p_{1}, q\right) \circ T_{1}=h\left(p_{1}, q\right)$ for each $q, 0 \leqq q<2^{p_{1}}$. There is a permutation $T_{2}$ of the intervals $I\left(p_{3}, s\right)$ leaving the intervals $I\left(p_{2}, q\right)$ invariant such that

$$
\begin{aligned}
c_{i} \psi_{r_{i}} \chi\left(p_{i}, q\right) \circ T_{2} \circ T_{1}=h\left(p_{i}, q\right) \\
0 \leqq q<2^{p_{i}}, \quad i=1,2, \quad c_{i}=2^{\left(p_{i-1) / 2}\right.} .
\end{aligned}
$$

By induction, there is a sequence $\left\{T_{n}\right\}$ of transformations of $I$ onto itself such that for each $n, T_{n}$ permutes the intervals $I\left(p_{n+1}, s\right)$ leaving invariant all intervals $I\left(p_{i}, q\right), i \leqq n$, and such that

$$
\begin{aligned}
c_{i} \psi_{r_{i}} \chi\left(p_{i}, q\right) \circ T_{n} \circ T_{n-1} \circ & \cdots \circ T_{1}=h\left(p_{i}, q\right) ; \\
0 & \leqq q<2^{p_{i}}, \quad i=1,2, \cdots, n .
\end{aligned}
$$

It is a standard fact that the sequence $\left\{T_{n}\right\}$ induces a measure preserving transformation $T$ of $I$ on to itself that is one-to-one except for a countable set of points. Clearly $c_{i} \psi_{r_{i}} \chi\left(p_{i}, q\right) \circ T=h\left(p_{i}, q\right)$; $0 \leqq q<2^{p_{i}}, i=1,2, \cdots$. Thus the span of the system $\left[\psi_{n_{j}} \circ T\right.$ : $\left.n_{j} \in S\right]$ contains a family of Haar functions $\left[h\left(p_{i}, q\right): 0 \leqq q<2 p_{i}\right.$, $\left.p_{1}<p_{2}<\cdots\right]$. This concludes the proof.

\section{REFERENCES}

1. N. J. Fine, On the Walsh functions, Trans. Amer. Math. Soc. 65 (1949), 372-414.

2. S. S. Kaczmarz and H. Steinhaus, Theorie der Orthogonalreihen, reprint, Chelsea, New York, 1951.

3. N. Levinson, Gap and density theorems, Amer. Math. Soc. Colloq. Publ., Vol. 26, Amer. Math. Soc., Providence, R. I., 1940.

4. J. J. Price and R. E. Zink, On sets of completeness for families of Haar functions, Trans. Amer. Math. Soc. 119 (1965), 262-269.

5. A. A. Talalyan, The representation of measurable functions by series, Uspehi Mat. Nauk 15 (1960), 77-142 = Russian Math. Surveys 15 (1960), 77-136. 